\title{
Another Form of Matrix Nim
}

\author{
Thomas S. Ferguson \\ Mathematics Department \\ UCLA, Los Angeles CA 90095, USA \\ tom@math.ucla.edu
}

Submitted: February 28, 2000; Accepted: February 6, 2001.

MR Subject Classifications: 91A46

\begin{abstract}
A new form of 2-dimensional nim is investigated. Positions are rectangular matrices of non-negative integers. Moves consist of chosing a positive integer and a row or column and subtracting the integer from every element of the chosen row or column. Last to move wins. The $2 \times 1$ case is just Wythoff's Game. The outcomes of all $2 \times 2$ positions are found in both the impartial and partizan cases. Some hope is given of being able to solve sums of $2 \times 2$ games in the partizan case.
\end{abstract}

\section{Introduction.}

There exist in the literature on combinatorial games several generalizations to two dimensions of the game of nim. The earliest is the game of matrix nim found in Holladay (1958). In this game, call it $N_{n}$, there is an $m \times n$ rectangular board of piles of counters. A move consists of either (1) removing any number of counters in the piles in one row, or (2) removing any number of counters from any of the piles provided one column is left untouched. Last to move wins. The game $N_{1}$ is the game nim.

In the Two-dimensional Nim found on page 313 of Winning Ways, Berlekamp et al. (1982), there are a finite number of counters in the non-negative integer lattice of the plane. A move consists of either (1) moving any counter to the left, or (2) moving any counter to any position in a lower row. When all counters are on the lowest row, the game is nim. This game is used to illustrate transfinite nim values.

In the Two-Dimensional Nim due to Eggleston, Fraenkel and Rothschild, found in Fraenkel (1994), a player may remove any number of counters from any row or any column. This is related to the game of Nimby usually played on a triangular board (see for example, Fraenkel and Herda (1980)), in which a move consists of removing any number of contiguous counters in an arbitrary line.

In the game of Shrage (1985), a position consists of a finite number of rectangles of integer sides. At each move, a rectangle is broken into two rectangles of unequal size, 
thus generalizing Grundy's game. And last but not least, there is Lenstra's remarkable extension to two dimensions of turning games in Chapter 14 of Winning Ways.

\section{The Rules of the Game.}

In this paper, another form of matrix nim is proposed. This form is also a generalization of Wythoff's Game, Wythoff (1908). The positions are $m \times n$ matrices of nonnegative integers where $m$ and $n$ are fixed positive integers. A move consists of choosing a row or column and subtracting some positive integer $k$ from each integer of the chosen row or column. The terminal positions are the matrices with at least one zero in every row and column. Last to move wins. The case $m=1$ and $n=2$ is Wythoff's Game, also called "Tsyan-shizi”.

The above is the impartial version of the game. There is also the partizan version in which Left is restricted to choosing a coLumn and Right is restricted to choosing a Row. The game remains unchanged if the rows are permuted; similarly for the columns. In addition, the impartial game remains unchanged if the matrix is transposed.

\section{A General Result.}

The complete game seems to be difficult to analyze. However, there is one general result that holds and is useful for playing square matrices. We say that an $n \times n$ square matrix $M=\left(a_{i j}\right)$ is diagonally subordinate if each off-diagonal element is at least as great as the sum of its two corresponding diagonal elements, i.e. $a_{i j} \geq a_{i i}+a_{j j}$ for all $i$ and $j, i \neq j$.

Theorem 1. In impartial or partizan $n \times n$ matrix nim, a diagonally subordinate matrix is a $P$-position (i.e. a second player win) if and only if its diagonal elements form a $P$-position in ordinary nim.

Proof. Any legal move in a diagonally subordinate matrix leads to another diagonally subordinate matrix. If the diagonal elements of the original matrix form a P-position in ordinary nim, they will not do so in any move from the position. Therefore there is a move back to a diagonally subordinate matrix whose diagonal elements do form a $\mathrm{P}$-position in ordinary nim. This move back to a P-position may be made either using a row or a column.

Elwyn Berlekamp (private communication, 1999) has suggested the following improvement of this theorem. Consider a general $m \times n$ matrix, $A$, and suppose there is some element $a_{i j}$ such that $a_{i j} \geq \min _{k} a_{k j}+\min _{\ell} a_{i \ell}$. Then $a_{i j}$ may be replaced in the matrix by any larger number without changing the value of the position. This is because any sequence of moves in the original game is also available in the modified game, and conversely. Since this is the case, we may replace such an $a_{i j}$ by the symbol $\infty$, which represents any number greater or equal to $\min _{k} a_{k j}+\min _{\ell} a_{i \ell}$. For example,

$$
\left(\begin{array}{cc}
10 & 5 \\
3 & 6
\end{array}\right) \quad \text { may be written } \quad\left(\begin{array}{cc}
\infty & 5 \\
3 & 6
\end{array}\right) \text {. }
$$


One may call a position reduced if one has replaced as many values as possible with infinities. A matrix may have several infinity entries, but there is at least one finite entry in every row and column. We may obtain the following theorem.

Theorem 2. If the matrix $A$ is block diagonal with infinities in all non-diagonal blocks, then the game (impartial or partizan) with matrix $A$ is equal to the sum of the games having as matrices the blocks on the diagonal.

For example,

$$
\left(\begin{array}{ccc}
17 & \infty & \infty \\
\infty & 7 & 5 \\
\infty & 6 & 8
\end{array}\right)=* 17+\left(\begin{array}{ll}
7 & 5 \\
6 & 8
\end{array}\right),
$$

in which $* n$ denotes as usual a nim pile of $n$ chips.

This theorem contains Theorem 1 for reduced matrices since the condition that the matrix be diagonally subordinate is equivalent to saying that all off-diagonal elements are $\infty$.

Proof. Let the blocks of $A$ be denoted by $B_{1}, \ldots, B_{r}$.

Let $A^{T}$, the transpose of $A$, denotes the game $A$ with the roles of the players reversed in the partizan case and the same game in the impartial case. We must show that the game

$$
A^{T}+B_{1}+\cdots+B_{r}
$$

is a second player win. This is done by a pairing argument. Any move in $A^{T}$ that intersects block $B_{i}^{T}$ is met by the corresponding move in $B_{i}$, and conversely. After such a move and its response, the game will again be of the form (3) so the second player will never be without a move.

\section{The Impartial $1 \times n$ Game.}

For $n=2$, the game is exactly Wythoff's Game.

For odd $n$, the P-positions are the same as for ordinary nim, namely those positions for which the nim-sum of the pile sizes is zero. This follows because the extra move of subtracting $k \geq 1$ from each pile changes the nim-sum of the piles. To see this, expand $k$ in binary and consider the least significant digit 1 . If $k$ is subtracted from any pile, the binary expansion for that pile size must change in that digit, and since there are an odd number of piles the nim-sum must change in that digit also. (In fact, if in nim we allow a player to remove the same number of counters from any odd number of piles of his choice, the P-positions are still those of nim.) Impartial $1 \times n$ nim with $n$ even is not nim.

The first difficult (still unsolved) case is $n=4$.

\section{Impartial $2 \times 2 \mathrm{Nim}$.}


The positions are $2 \times 2$ matrices, $M=\left(\begin{array}{ll}a & b \\ c & d\end{array}\right)$, with nonnegative integer entries. Theorem 1 can be strengthened in the impartial case.

Lemma 1. For $a \geq b$ and $d \geq b,\left(\begin{array}{ll}a & b \\ b & d\end{array}\right)$ is a P-position.

Proof. Any move subtracting $k$ from some row (column), can be countered by subtracting $k$ from the other row (column).

This lemma is false in the partizan case, e.g. $\left(\begin{array}{ll}1 & 1 \\ 1 & 2\end{array}\right)= \pm 1$ is an N-position.

Note that the outcome of a position is not changed if the matrix is rotated 90 degrees. We show that all $\mathrm{P}$-positions for $2 \times 2$ nim have at least one constant diagonal. Specifically,

Theorem 3. $M$ is a $P$-position if and only if a rotation can put $M$ into one of the following two types.

$$
\left(\begin{array}{ll}
a & b \\
b & d
\end{array}\right) \quad \text { with } a \geq b \text { and } d \geq b
$$

and

$$
\left(\begin{array}{ll}
a & b \\
b & d
\end{array}\right) \quad \text { with } a<b<d \text { and } a \bmod (d-a)<d-b
$$

Example. If the initial position is $\left(\begin{array}{ll}20 & 24 \\ 24 & 27\end{array}\right)$, then although it has constant diagonal with $a<b<d$, it is not $\mathrm{P}$ since $20 \bmod (27-20)=6$ which is greater than $27-24=$ 3. Thus it is a first player win and the only winning move is to remove 7 from the components of the last row or column, moving to $\left(\begin{array}{ll}17 & 20 \\ 20 & 24\end{array}\right)$, which satisfies condition (5) since $17 \bmod (24-17)=3$ is now $<(24-20)=4$ and so is a P-position.

To prove Theorem 3, the following lemma is useful. It shows that what appeared in the example is true in general: that if a position with constant diagonal is moved to another position with constant diagonal, then one and only one of the two positions will satisfy (5).

Lemma 2. Suppose $0 \leq a<b<d \leq a+b$. Let $a^{\prime}=a+b-d, b^{\prime}=a$ and $d^{\prime}=b$. Then $0 \leq a^{\prime}<b^{\prime}<d^{\prime}$ and

$$
a \bmod (d-a)<d-b \quad \text { if and only if } \quad a^{\prime} \bmod \left(d^{\prime}-a^{\prime}\right) \geq d^{\prime}-b^{\prime} .
$$

Proof. Note that $d^{\prime}-a^{\prime}=d-a$. Let $r=a \bmod (d-a)$ and $s=a^{\prime} \bmod (d-a)$. Then $s \equiv r+b-d \equiv r+b-a \quad(\bmod d-a)$. If $0 \leq r<d-b$, then $b-a \leq r+b-a<d-a$ 
so that $s=r+b-a$, and $s \geq b-a$. Conversely, if $b-a \leq s<d-a$, then $r=s-(b-a)$ $(\bmod d-a)$ and $0 \leq s-(b-a)<d-b$, so that $r=s-(b-a)<d-b$.

Proof of Theorem 3. The terminal positions all satisfy (4) with $b=0$. Lemma 1 says that all positions satisfying (4) are P-positions. It is easy to see that one cannot move a position satisfying (4) into a position satisfying (5). To complete the proof, we must show

(i) every follower of a position satisfying (5) does not satisfy (4) or (5), and

(ii) every position not satisfying (4) or (5) has a follower satisfying (4) or (5).

(i) Suppose $M$ is of the form $\left(\begin{array}{ll}a & b \\ b & d\end{array}\right)$ with $a \leq d$. If $a=d$ or if $a<d$ and $d>a+b$, then $M$ cannot be moved to constant diagonal form. Otherwise, $a<d \leq a+b$ and there is a unique move of $M$ to constant diagonal form by removing $d-a$ from the last row or column. This moves to say $\left(\begin{array}{cc}a & b-d+a \\ b & a\end{array}\right)$, a matrix equivalent to $\Delta=\left(\begin{array}{cc}a^{\prime} & b^{\prime} \\ b^{\prime} & d^{\prime}\end{array}\right)$, where $a^{\prime}=a+b-d, b^{\prime}=a$ and $d^{\prime}=b$.

First note that $\Delta$ does not satisfy (4) since $a^{\prime}=a+b-d<a=b^{\prime}$.

Since $b^{\prime}<d^{\prime}$ (this is $a<b$ and so is true), the only if part of Lemma 2 implies that $\Delta$ cannot satisfy (5). So far so good.

(ii) Suppose $M$ is constant diagonal but does not satisfy (4) or (5). There are two possibilities for matrices $\left(\begin{array}{ll}a & b \\ b & d\end{array}\right)$ for $a \leq d$. First it may be that $a<b$ and $d \leq b$. Then the move to $\left(\begin{array}{cc}a & b \\ b-d+a & a\end{array}\right)$ moves to a position satisfying (4). Otherwise, $a<b<d$ and $a \bmod (d-a) \geq d-b$. A move to constant diagonal form as in part (i) must be to $\Delta$ as given there. But now the if part of Lemma 2 shows that $\Delta$ satisfies (5).

Now suppose that $M=\left(\begin{array}{ll}a & b \\ c & d\end{array}\right)$ is not of diagonal form. Suppose without loss of generality that $a \leq b<c$ and $a<d$.

If $a=b$, then the lower row may be reduced so that the minimum value of that row is equal to $a$. This moves to a position of form (4). So we may assume $a<b$.

If $d \leq c$, then the lower row may be reduced by $d-a$, again a move to form (4). So we may assume $a<b<c<d$.

There may be up to four moves from such a matrix $M$ to constant diagonal form. However, there are at most two moves to constant diagonal form that involve the last row. We will show that one of these is a move to form (5).

One such move is to $\Delta_{1}=\left(\begin{array}{cc}a & b \\ b & b+d-c\end{array}\right)$. This move may always be made. The other move is to $\left(\begin{array}{cc}a & b \\ a+c-d & a\end{array}\right)$, a move which is possible if $a+c \geq d$. This matrix is 
equivalent to $\Delta_{2}=\left(\begin{array}{ll}a^{\prime} & b^{\prime} \\ b^{\prime} & d^{\prime}\end{array}\right)$, where $a^{\prime}=a+c-d, b^{\prime}=a$ and $d^{\prime}=b$. Both $\Delta_{1}$ and $\Delta_{2}$ satisfy the first requirement of satisfying (5), namely $a^{\prime}<b^{\prime}<d^{\prime}$.

If $\Delta_{1}$ satisfies (5), we are finished. If not, then $a \bmod (b+d-c-a) \geq(d+b-c)-b=$ $d-c$, and the move to $\Delta_{2}$ is possible since $a \geq a \bmod (b+d-c-a) \geq d-c$ implies $a+c \geq d$. But then by Lemma 2 again, $\Delta_{2}$ must satisfy (5).

It is interesting to note that from any N-position of the form $\left(\begin{array}{ll}a & b \\ c & d\end{array}\right)$ with $a \leq b \leq$ $c$ and $a \leq d$, there exists a move to a P-position using the bottom row. Sprague-Grundy values for Wythoff's Game are apparently chaotic (see Winning Ways, vol. 1, p. 76). One suspects the same is true for this game.

\section{Partizan $2 \times 2$ Nim.}

The corresponding result for partizan $2 \times 2$ nim is somewhat simpler than for the impartial case. There are four outcome classes in partizan games, the P-positions that the Previous player wins, the N-positions that the Next player wins, the R-positions that Right wins no matter who plays first, and the L-positions that Left wins no matter who goes first.

Theorem 4. By interchanging rows or interchanging columns if necessary, consider only positions of the form $\left(\begin{array}{ll}a & b \\ c & d\end{array}\right)$ with $a=\min \{a, b, c, d\}$. Then all L-positions are of the form,

$$
a+c<\min \{b, d\} .
$$

All R-positions are of the form,

$$
a+b<\min \{c, d\}
$$

All P-positions are of the form,

$$
(a=d \quad \text { and } \quad \min \{b, c\} \geq a+d) \quad \text { or } \quad(a=0 \quad \text { and } \quad b=c<d)
$$

Everything else is an $\mathrm{N}$-position.

Proof. It is easy to show that positions of the form (9) are all P-positions, positions of the form (8) are all R-positions, and positions of the form (7) are all L-positions. Therefore, it is sufficient to show that from every other position, Right can move either to $\mathrm{P}$ or to $\mathrm{R}$, since then by symmetry, Left can move to $\mathrm{P}$ or $\mathrm{L}$.

If $a=0$, then a position does not satisfy (7), (8) or (9) if and only if $d \leq b$ and $d \leq c$. Right may move such a position into $\mathrm{P}$ by removing $d$ from the bottom row. So we may now assume $a>0$. 
Moves that take as much as possible and leave a 0 in some entry of the matrix are very powerful. If we take $a$ from the top row, the result satisfies (8) if and only if $b-a<\min \{c, d\}$. If we take $\min \{c, d\}$ from the bottom row, the result satisfies (8) if and only if $|c-d|<a$. Suppose a position cannot be moved into $\mathrm{R}$ by this method, and is not in L. Then

$$
|c-d| \geq a, \quad b-a \geq \min \{c, d\}, \quad \text { and } \quad a+c \geq \min \{b, d\} .
$$

We will be finished if we can show that Right can move such a position into $\mathrm{P}$ unless it is already in $\mathrm{P}$.

First suppose $c<d$. Then from (10), $d \geq a+c, b \geq a+c$, and $a+c \geq \min \{b, d\}$. This implies $a+c=\min \{b, d\}$. If $a+c=d \leq b$, then taking $c$ from the bottom row moves to $\left(\begin{array}{ll}a & b \\ 0 & a\end{array}\right)$ which is in P. If $a+c=b<d$, then taking $a$ from the top row moves to $\left(\begin{array}{ll}0 & c \\ c & d\end{array}\right)$ which is in $\mathrm{P}$.

Suppose now that $c \geq d$. Then from (10), $c \geq a+d$, and $b \geq a+d$. This is diagonally subordinate and so can be moved to $\mathrm{P}$ if not already in $\mathrm{P}$.

\section{An Investigation into Values of Partizan $2 \times 2 \mathrm{Nim}$.}

It may be possible to find a method of solving a sum of $2 \times 2$ partizan nim games.

Here is a result for positions with $a=0$. Note that since $\left(\begin{array}{ll}a & b \\ c & d\end{array}\right)=-\left(\begin{array}{ll}a & c \\ b & d\end{array}\right)$, we may assume $b \geq c$ without loss of generality.

Theorem 5. Let $v$ denote the value of $\left(\begin{array}{ll}0 & b \\ c & d\end{array}\right)$ and assume $b \geq c$. Then

1. If $d \leq c$, then $v=* d$.

2. If $b=c$ and $c<d$, then $v=0$.

3. If $b>c$ and $c<d \leq 2 c$, then $v=2^{-(2 c+1-d)}$.

4. If $b>c$ and $2 c<d$, then $v=\min \{d-2 c, b-c\}$.

Proof. Part 1 follows from Theorem 1. Part 2 follows from Theorem 4. If $c=0$ then clearly, $v=\min \{b, d\}$. So assume $0<c<b$ and $c<d$. We prove Parts 3 and 4 together by induction on the sum of the components.

The right options of $\left(\begin{array}{ll}0 & b \\ c & d\end{array}\right)$ are $\left(\begin{array}{cc}0 & b \\ c-k & d-k\end{array}\right)$ for $k=1, \ldots, c$. By induction,

$$
\left(\begin{array}{cc}
0 & b \\
c-k & d-k
\end{array}\right)= \begin{cases}2^{-(2 c+1-d-k)} & \text { if } k \leq 2 c-d \\
\min \{d-2 c, b-c\}+k & \text { if } k>2 c-d\end{cases}
$$


These are all numbers, increasing in $k$, so domination reduces the options to the single option with $k=1$.

The left options of $\left(\begin{array}{ll}0 & b \\ c & d\end{array}\right)$ are $\left(\begin{array}{ll}0 & b-k \\ c & d-k\end{array}\right)$ for $k=1, \ldots, \min \{b, d\}$. If $b \geq d$, then by induction,

$$
\left(\begin{array}{ll}
0 & b-k \\
c & d-k
\end{array}\right)= \begin{cases}\min \{d-2 c, b-c\}-k & \text { if } k<d-2 c \\
2^{-(2 c+1-d+k)} & \text { if } d-2 c \leq k<d-c \\
*(d-k) & \text { if } k \geq d-c .\end{cases}
$$

The numbers are decreasing in $k$, and positive. If $d>c+1$, there is at least one positive number, and the option $k=1$ dominates the others. Otherwise (if $d=c+1$ ), the options are the nimbers, $* c, *(c-1), \ldots, 0$. But the right options are all positive numbers, so the nimbers, $* c, \ldots, * 1$ are all reversible through 0 , and so may be removed. In this case, left's options are reduced to the option $k=d$.

If $b<d$, then it may similarly be shown that all left options, $\left(\begin{array}{cc}0 & b-k \\ c & d-k\end{array}\right)$, are numbers, decreasing in $k$ (though some may be negative). Thus the option $k=1$ dominates the others.

Putting this together, we find that for the special case $d=c+1$,

$$
\left(\begin{array}{ll}
0 & b \\
c & d
\end{array}\right)=\left\{\left(\begin{array}{cc}
0 & b-d \\
c & 0
\end{array}\right) \mid\left(\begin{array}{cc}
0 & b \\
c-1 & d-1
\end{array}\right)\right\}=\left\{0 \mid 2^{-(2 c-d)}\right\}=2^{-(2 c-d+1)}
$$

agreeing with Part 3 since $d=c+1<2 c$. In all other cases, $(0<c<b, d>c+1)$,

$$
\left(\begin{array}{ll}
0 & b \\
c & d
\end{array}\right)=\left\{\left(\begin{array}{ll}
0 & b-1 \\
c & d-1
\end{array}\right) \mid\left(\begin{array}{cc}
0 & b \\
c-1 & d-1
\end{array}\right)\right\}
$$

In these cases, we find

$$
\left(\begin{array}{ll}
0 & b-1 \\
c & d-1
\end{array}\right)= \begin{cases}0 & \text { if } b=c+1 \\
2^{-(2 c-d+2)} & \text { if } b>c+1 \text { and } d \leq 2 c+1 \\
\min \{d-2 c, b-c\}-1 & \text { if } b>c+1 \text { and } d>2 c+1\end{cases}
$$

and

$$
\left(\begin{array}{cc}
0 & b \\
c-1 & d-1
\end{array}\right)= \begin{cases}2^{-(2 c-d)} & \text { if } d \leq 2 c-1 \\
\min \{d-2 c, b-c\}+1 & \text { if } d>2 c-1\end{cases}
$$

In all these cases, it is straightforward to check that Parts 3 and 4 hold as well.

All of the positions with $a=0$ are either nimbers or numbers. However, most of the positions for $\min \{a, b, c, d\}>0$ are hot, and many are switches. (A switch is a position of the form $\{a \mid b\}$ with $a>b$.) The simplest example is $\left(\begin{array}{ll}1 & 1 \\ 1 & 1\end{array}\right)= \pm 1$. 
Conjecture: For $a<c$ and for $b$ and $d$ sufficiently large (say, $d>2 c$ and $b>a+c$ ), we have $v=\min \{b-c, a+d-2 c\} \pm a$. From such a position, Left can move to $\left(\begin{array}{cc}0 & b \\ c-a & d\end{array}\right)$ which has value $\min \{b-c, a+d-2 c\}+a$ from Theorem 5 , while right can move to $\left(\begin{array}{cc}0 & b-a \\ c & d\end{array}\right)$ with value $\min \{b-c, a+d-2 c\}-a$.

Counterexample. $\left(\begin{array}{ll}1 & 2 \\ 2 & 2\end{array}\right)$ is the simplest position that is not a number, nor a nimber nor a switch. Here is an example to show that it is not necessarily best to move to a matrix with at least one 0 . In the symmetric game, $\left(\begin{array}{ll}1 & 2 \\ 2 & 2\end{array}\right)+\left(\begin{array}{ll}1 & 1 \\ 1 & 1\end{array}\right)$, Left's only winning move is to $\left(\begin{array}{ll}1 & 1 \\ 2 & 1\end{array}\right)+\left(\begin{array}{ll}1 & 1 \\ 1 & 1\end{array}\right)$.

Acknowledgement. I would like to thank the referees for their careful reading and useful suggestions.

\section{References}

E. R. Berlekamp, J. H. Conway and R. K. Guy (1982) Winning Ways for Your Mathematical Plays, in two volumes, Academic Press, New York.

Aviezri Fraenkel (1994) Combinatorial Games, in The Lighter Side of Mathematics, R. K. Guy and R. E. Woodrow, eds., Mathematical Association of America, 176-194

A. S. Fraenkel and H. Herda (1980) Never rush to be first in playing Nimbi, Math. Mag. 53, 21-26.

J. C. Holladay (1958) Matrix nim, Amer. Math. Mo. 65, 107-109.

G. Schrage (1985) A two-dimensional generalization of Grundy's game, Fibonacci Quart. 23, 325-329.

W. A. Wythoff (1907) A modification of the game of nim, Nieuw Arch. Wisk. 7, 199-202. 